\title{
Diffusion Behaviors of Hydrogen Isotopes in Incoloy 800H: A First-Principles Study
}

\author{
Hongyu Chen, ${ }^{1,2,3}$ Hong Li, ${ }^{1,2,3}$ Chuan Li, ${ }^{1,2,3}$ Jianzhu Cao, ${ }^{1,2,3}$ and Chao Fang ${ }^{1,2,3}$ \\ ${ }^{1}$ Institute of Nuclear and New Energy Technology, Tsinghua University, Beijing 100084, China \\ ${ }^{2}$ Collaborative Innovation Center of Advanced Nuclear Energy Technology, Beijing 100084, China \\ ${ }^{3}$ The Key Laboratory of Advanced Reactor Engineering and Safety, Ministry of Education, Beijing 100084, China
}

Correspondence should be addressed to Chao Fang; fangchao@tsinghua.edu.cn

Received 24 May 2017; Revised 23 August 2017; Accepted 11 September 2017; Published 10 October 2017

Academic Editor: Michael I. Ojovan

Copyright (C) 2017 Hongyu Chen et al. This is an open access article distributed under the Creative Commons Attribution License, which permits unrestricted use, distribution, and reproduction in any medium, provided the original work is properly cited.

\begin{abstract}
Incoloy $800 \mathrm{H}$ is one of the main stainless steel materials used in steam generators with High Temperature Reactor Pebble-bed Modules (HTR-PM). In this study, the diffusion behaviors of hydrogen isotopes in Incoloy $800 \mathrm{H}$ were investigated with firstprinciple calculations. Numerical results reveal that the starting and ending positions of the diffusion process are the two adjacent and most stable octahedral sites surrounded by Fe atoms and $\mathrm{Ni}$ atoms, and the diffusion follows an indirect path via the metastable tetrahedral sites and octahedral sites surrounded by $\mathrm{Fe}$ atoms and $\mathrm{Cr}$ atoms. The diffusion activation energies of hydrogen $(\mathrm{H})$, deuterium (D), and tritium (T) in Incoloy $800 \mathrm{H}$ are investigated by first-principles calculations with the same approximate value of $Q=0.757 \mathrm{eV}$; the diffusion coefficient frequency factors are also obtained with values of $D_{0}=1.56 \times 10^{-6}, 1.10 \times 10^{-6}$, and $8.99 \times 10^{-7}\left(\mathrm{~m}^{2} / \mathrm{s}\right)$ for $\mathrm{H}, \mathrm{D}$, and T, respectively. Furthermore, the theoretical results are compared with the experimental data, and it is found that both are in agreement with each other. These results are very helpful for understanding the diffusion behaviors of hydrogen isotopes in Incoloy $800 \mathrm{H}$ and can be used to guide the tritium source term analysis of secondary circuits in HTR-PM, which are first studied from a microperspective.
\end{abstract}

\section{Introduction}

Tritium is one of the most important radionuclides in reactors owing to its very harmful $\beta$-radiation and long half-life. Although the $\beta$-particle produced by the decay of tritium is easily absorbed and blocked by the skin, tritium oxide ( $\mathrm{HTO}, \mathrm{DTO}$, or $\mathrm{T}_{2} \mathrm{O}$ ) can be inhaled and can combine with organic matter, resulting in internal irradiation, which is very harmful to the human body. In the primary circuit of High Temperature Reactor Pebble-bed Modules (HTR-PM), tritium could be generated in two ways: ternary fission and neutron reactions [1]. Additionally, the experimental results have indicated that tritium diffusion is a serious issue for High Temperature Gas-cooled Reactors (HTGR) because tritium could easily traverse metal and permeate from the primary circuit into the secondary circuit under high temperature conditions [2]. It is reported that, at the end of the 20-year lifetime of a 10 MW High Temperature Gas-cooled Test Reactor
(HTR-10), the radioactivity of ${ }^{3} \mathrm{H}$ in the primary circuit will have accumulated to $5.7 \times 10^{9} \mathrm{~Bq}$, and it is estimated that the concentration of ${ }^{3} \mathrm{H}$ in the secondary circuit of a $1000 \mathrm{MWe}$ HTGR could be $1.85 \times 10^{5} \mathrm{~Bq} / \mathrm{L}$ in the equilibrium state $[1,3]$. The steam generator pipes are the main boundary for tritium permeation, and Incoloy $800 \mathrm{H}$ is a major material of those pipes in HTR-PM, which makes it essential to understand the diffusion mechanism of tritium in Incoloy $800 \mathrm{H}$.

In recent years, a number of theoretical studies based on first-principles calculations of diffusion mechanisms and behaviors of gas atoms in metal have been published [4-9]. Wimmer et al. have predicted the temperature-dependent diffusion coefficients of interstitial hydrogen, deuterium, and tritium atoms in nickel [4]. Yang et al. studied the diffusion behaviors of $\mathrm{C}, \mathrm{N}$, and $\mathrm{O}$ atoms in $\mathrm{V}$ metal [5]. Liu et al. have researched the diffusion activation energies of $\mathrm{C}, \mathrm{N}$, and $\mathrm{O}$ in $\alpha$-Fe [6]. Apart from these theoretical studies, many experimental measurements on diffusion have been 


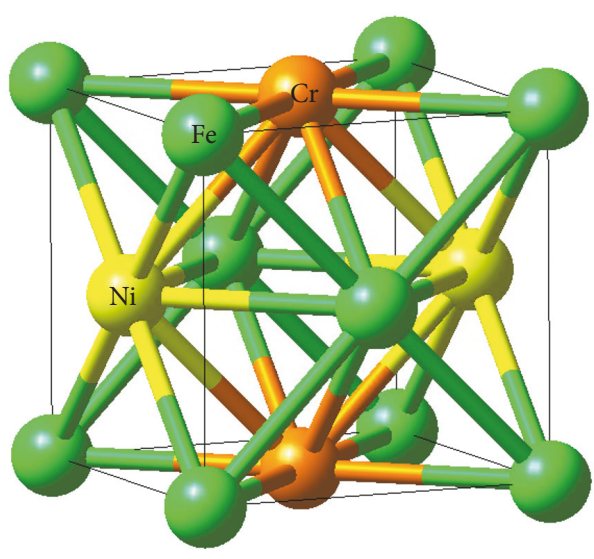

(a)

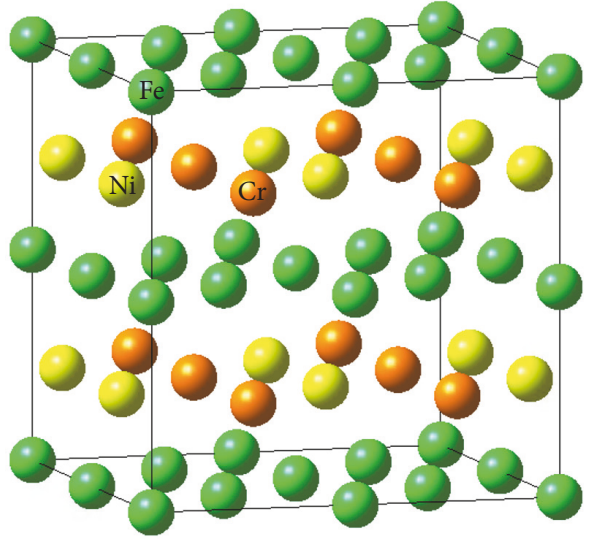

(b)

Figure 1: (a) The cell of face-centered cubic iron with two Fe atoms replaced by a Cr atom and an Ni atom. (b) $2 \times 2 \times 2$ face-centered cubic supercell. In all figures, green atoms represent $\mathrm{Fe}$, yellow $\mathrm{Ni}$ atoms, and orange $\mathrm{Cr}$ atoms.

performed, proving that the first-principle calculation is a powerful research tool in materials science [10-14].

However, there are few studies on the diffusion of gas atoms in Incoloy $800 \mathrm{H}$ and other alloy materials. In this study, the diffusion mechanism and behavior of hydrogen isotopes $(\mathrm{H}, \mathrm{D}$, and $\mathrm{T}$ ) in Incoloy $800 \mathrm{H}$ were investigated with first-principle calculations using density functional theory (DFT) [15]. In addition, the numerical results of the calculation, including the diffusion activation energies and the frequency factors of diffusion coefficients, were compared with the experimental results of hydrogen and deuterium obtained by our team. The similarity between these results shows that the study is reliable and sometimes, in nuclear industry, the first-principle calculation could take the place of complicated experiments in rigorous conditions, saving costs and resources.

\section{Method of Calculation and Experiment}

2.1. The Construction of Incoloy $800 \mathrm{H}$ Model. The calculation was performed based on DFT as implemented in the Vienna Ab initio Simulation Package (VSAP) with the projector augmented wave (PAW) potential method [16-19]. The exchange and correlation effects are described by the generalized gradient approximation (GGA) in the PerdewBurke-Ernzerhof (PBE) form [20]. To carry out this study, the microstructure of Incoloy $800 \mathrm{H}$ has to be determined in the first step.

Incoloy $800 \mathrm{H}$ is an austenitic alloy with a face-centered cubic structure. Its main components are $\mathrm{Fe}, \mathrm{Cr}$, and $\mathrm{Ni}$ with the mass fractions of approximately $46 \%, 32 \%$, and $21 \%$, respectively [21]. The numerical ratio of these three elements can be easily calculated as being approximately $6: 4: 3$. This ratio is similar to that obtained on replacing two iron atoms by one chromium atom and one nickel atom in each ideal primitive cell of face-centered cubic iron (Figure 1(a)), and, therefore, the ratio can be simplified to $2: 1: 1$. Finally, the system is constructed using a $2 \times 2 \times 2$ supercell containing
$16 \mathrm{Fe}$ atoms, $8 \mathrm{Cr}$ atoms, and $8 \mathrm{Ni}$ atoms (Figure $1(\mathrm{~b})$ ). To check the convergence of the activation energies, we have also considered a $3 \times 3 \times 3$ supercell containing 72 host atoms and one hydrogen atom. The lattice constant was optimized to $3.486 \AA$, the calculation was performed by using a $5 \times 5 \times$ $5 k$-point mesh, and the plane wave cutoff was $500 \mathrm{eV}$.

\subsection{Diffusion Activation Energy and Diffusion Coefficient.} The most common diffusion mechanism of hydrogen isotopes in crystalline solids is via the vacancies composed of lattice atoms [8]. There are two types of conventional vacancy trap sites in face-centered cubic cells: octahedral sites and tetrahedral sites. In addition, owing to the differences in vicinal atoms, the octahedral sites fall into three types, while there is only one type of tetrahedral site (Figure 2).

The general form of the diffusion coefficient is given by

$$
D=D_{0} \times \exp \left(-\frac{Q}{k_{B} T}\right),
$$

where $D_{0}$ is the temperature independent frequency factor, $k_{B}$ is the Boltzmann constant, and $T$ is the temperature. $Q$ is the diffusion activation energy, that is, the highest energy barrier for the impurity atoms to diffuse from one most stable vacancy to another [5].

According to the transition state theory, the transition rate of atoms in solids is given by [22]

$$
v=v \cdot \exp \left(-\frac{Q}{k_{B} T}\right),
$$

where $v$ is the vibrational frequency of the impurity. Therefore, the diffusion coefficient can be expressed as [23]

$$
D=l^{2} v \exp \left(-\frac{Q}{k_{B} T}\right),
$$




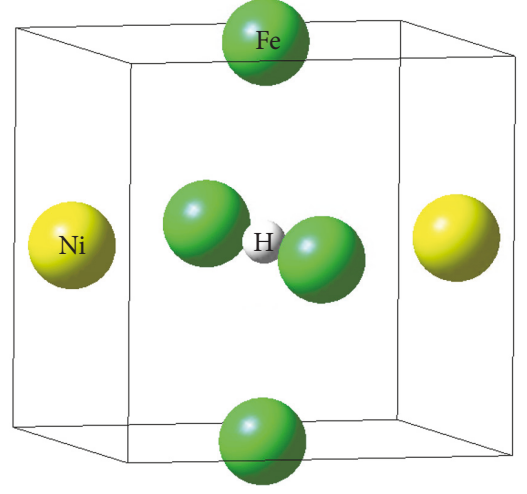

(a)

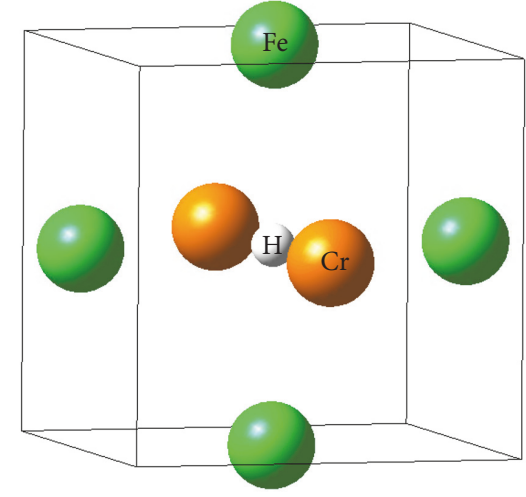

(b)

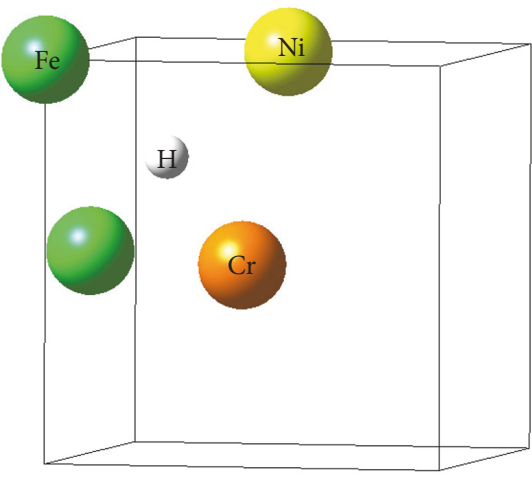

(d)

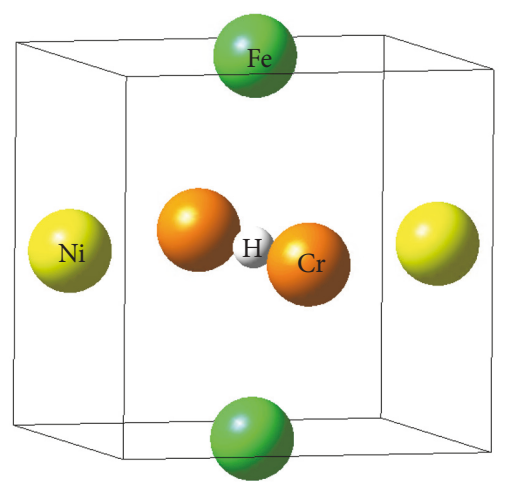

(c)

Figure 2: $(\mathrm{a}-\mathrm{c})$ The octahedral site surrounded by different base atoms. (a) The adjacent atoms are Fe and Ni (O-FeNi). (b) The adjacent atoms are $\mathrm{Fe}$ and $\mathrm{Cr}(\mathrm{O}-\mathrm{FeCr})$. (c) The adjacent atoms are $\mathrm{Fe}, \mathrm{Cr}$, and $\mathrm{Ni}(\mathrm{O}-\mathrm{FeCrNi})$. (d) The single tetrahedral site (Te). In all figures, white atoms represent $\mathrm{H}$ impurities. To represent the vacancy site more clearly, only the adjacent atoms are shown in these figures.

where $l$ is the length of every transition. Using Zener and Wert's theory, the vibrational frequency can be defined as $[24,25]$

$$
v=\sqrt{\frac{Q}{2 m l^{2}}},
$$

where $m$ is the mass of the impurity. Substituting (3) and (4) into (1), $D_{0}$ and $D$ can be written as

$$
\begin{aligned}
D_{0} & =\sqrt{\frac{Q l^{2}}{2 m}}, \\
D & =\sqrt{\frac{Q l^{2}}{2 m}} \exp \left(-\frac{Q}{k_{B} T}\right) .
\end{aligned}
$$

It is clear from the above derivation that the main challenge of the study is to determine the diffusion path and quantify the parameters $Q$ and $l$.

\section{Results and Discussion}

3.1. Diffusion Activation Energy. To confirm the physical diffusion path, the most stable vacancy site should be found
TABLE 1: System energy of $\mathrm{H}$ atom located in the four sites for $2 \times 2 \times 2$ and $3 \times 3 \times 3$ supercell models, respectively.

\begin{tabular}{lccccc}
\hline Vacancy sites & & O-FeCr & O-FeNi & O-FeCrNi & Te \\
\hline \multirow{3}{*}{$2 \times 2 \times 2$} & $E_{\mathrm{s}}(\mathrm{eV})$ & -251.9 & -251.7 & -251.6 & -251.3 \\
& & 37 & 85 & 41 & 21 \\
& $\Delta E_{\mathrm{s}}(\mathrm{eV})$ & - & 0.152 & 0.296 & 0.616 \\
\hline \multirow{3}{*}{$3 \times 3 \times 3$} & $E_{\mathrm{s}}(\mathrm{eV})$ & -777.9 & -777.9 & -777.7 & -777.3 \\
& $\Delta E_{\mathrm{s}}(\mathrm{eV})$ & - & 08 & 90 & 21 \\
& & & 0.028 & 0.146 & 0.615 \\
\hline
\end{tabular}

in the first step. The system energy $E_{s}$ of $H$ located in the three octahedral sites and the tetrahedral site was calculated (Table 1).

In this calculation with VASP, the projector augmented wave (PAW) pseudopotential was employed to describe the electron-ion interaction while neutron effects and mass effects were not involved. Therefore, the energies of $\mathrm{H}, \mathrm{D}$, and $\mathrm{T}$ should be the same; this is confirmed by our calculation. 


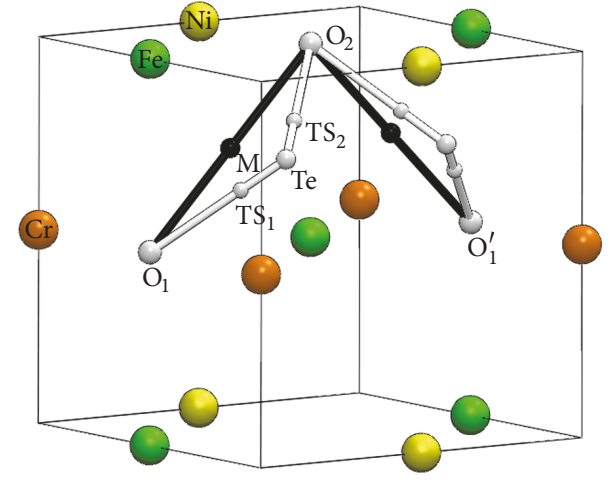

Figure 3: Two possible paths of one impurity atom diffusing from an $\mathrm{O}-\mathrm{FeCr}$ site $\mathrm{O}_{1}$ to another $\mathrm{O}-\mathrm{FeCr}$ site $\mathrm{O}_{1}^{\prime}$. $\mathrm{O}_{2}$ is an $\mathrm{O}$-FeNi site. Te denotes a tetrahedral interstitial site.

This is usually well justified as the barrier for an atomic jump is determined by the electronic interaction, which is identical for two isotopes of the same element, and not by the masses of the nuclei [25].

It is found that the most stable site is the $\mathrm{O}-\mathrm{FeCr}$ site where $E_{\mathrm{s}}$ equals to $-251.937 \mathrm{eV}$, lower than the Te site with a $2 \times 2 \times 2$ supercell by $0.616 \mathrm{eV}$. The $3 \times 3 \times 3$ supercell gives the same order of $E_{\mathrm{s}}$ of the four sites and an essentially same energy difference of $0.615 \mathrm{eV}$. Therefore, the impurity atoms must transit through an $\mathrm{O}-\mathrm{FeNi}$ site or an $\mathrm{O}-\mathrm{FeCrNi}$ site when they complete a whole diffusion process from one $\mathrm{O}-\mathrm{FeCr}$ site to another. Owing to the lower $E_{s}$, the OFeNi site is considered as the midpoint. As the starting, end, and midpoints were confirmed, there are two possible diffusion paths in the model, as illustrated in Figure 3. In path $\mathrm{A}, \mathrm{a} \mathrm{H}$ atom transits from $\mathrm{O}_{1}$ to $\mathrm{O}_{2}$ in a straight line; in path $\mathrm{B}$, the impurity atom jumps by a broken line, passing by the Te position. To compare the two diffusion paths, the "Transition State Search" method, nudged elastic band calculation [26], was used to search for the transition state.

As shown in Figure 4, the highest energy barriers (diffusion barriers) of path $\mathrm{A}$ and $\mathrm{B}$ are $1.152 \mathrm{eV}$ and $0.757 \mathrm{eV}$, respectively. It is obvious that impurity atoms tend to diffuse through path $\mathrm{B}$ owing to the lower energy barrier, and its diffusion activation energy $Q$ is $0.757 \mathrm{eV}$. The resulting energy curves also show that the energy at the tetrahedral sites is a local minimum of path B. Thus, the diffusion atoms would pass through the metastable tetrahedral sites acting as a transition state.

An experiment testing hydrogen diffusion performance at medium-high temperatures by gas-phase hydrogen permeation methods was also conducted.

As it is illustrated in Figure 5, an Incoloy $800 \mathrm{H}$ sample tube was maintained under high vacuum and several experimental temperatures. Hydrogen flowed from its source to inside the tube and then diffused outwards driven by the concentration difference. A quadrupole mass spectrometer was used to measure hydrogen concentration.
TABLE 2: Comparison between first-principles and experimental diffusion activation energy $Q$.

\begin{tabular}{lccc}
\hline Studies & $Q_{\mathrm{H}}(\mathrm{eV})$ & $Q_{\mathrm{D}}(\mathrm{eV})$ & $T\left({ }^{\circ} \mathrm{C}\right)$ \\
\hline Calculations & 0.757 & 0.757 & - \\
Experimental [15] & $0.493 \pm 0.037$ & $0.497 \pm 0.036$ & $300-550$ \\
$\eta$ & $53.55 \%$ & $52.31 \%$ & - \\
\hline
\end{tabular}

TABLE 3: The calculation and experimental results of temperature independent preexponential factor $D_{0}$.

\begin{tabular}{lccc}
\hline Studies & $\begin{array}{c}D_{0}(\mathrm{H}) \\
\left(\mathrm{m}^{2} / \mathrm{s}\right)\end{array}$ & $\begin{array}{c}D_{0}(\mathrm{D}) \\
\left(\mathrm{m}^{2} / \mathrm{s}\right)\end{array}$ & $\begin{array}{c}D_{0}(\mathrm{~T}) \\
\left(\mathrm{m}^{2} / \mathrm{s}\right)\end{array}$ \\
\hline Calculation & $1.56 \times 10^{-6}$ & $1.10 \times 10^{-6}$ & $8.99 \times 10^{-7}$ \\
Experimental & $6.31 \times 10^{-7}$ & $5.19 \times 10^{-7}$ & - \\
$\eta$ & $147.23 \%$ & $115.76 \%$ & - \\
\hline
\end{tabular}

According to the measured hydrogen permeation curve, the temperature-dependent diffusion coefficient $D(T)$ was calculated by the lag time method:

$$
D(T)=\frac{d^{2}}{6 t},
$$

where $d$ is the thickness of the sample tube and $t$ is the characteristic time, that is, the time for the hydrogen permeation to reach 0.617 times the saturated permeability.

The experimental data can be fitted with the logarithmic form of the Arrhenius relation, and the activation energy results are listed in Table 2 with their corresponding theoretical values. Considering the parameter difference between experimental and theoretical approach (such as temperature, pressure, gas concentration, and the structural defects of experimental material), this deviation is acceptable. In addition, since the values of $Q_{H}$ and $Q_{D}$ are similar to each other in experiment, it is reasonable to infer that the value of $Q_{T}$ would also be close to $Q_{H}$ and $Q_{D}$.

3.2. Diffusion Coefficients. As is shown in Figure 4, the length of every transition $l$ is $2.584 \AA\left(\mathrm{O}_{1}\right.$ to $\left.\mathrm{O}_{2}\right)$. Substituting $Q$ and $l$ to (5), $D_{0}$ of $\mathrm{H}, \mathrm{D}$, and $\mathrm{T}$ are shown in Table 3.

Finally, the complete diffusion coefficients of $\mathrm{H}, \mathrm{D}$, and $\mathrm{T}$ can be written as

$$
\begin{aligned}
& D(\mathrm{H})=1.56 \times 10^{-6} \times \exp \left(-\frac{0.757 \mathrm{eV}}{k_{B} T}\right) \mathrm{m}^{2} \cdot \mathrm{s}^{-1} \\
& D(\mathrm{D})=1.10 \times 10^{-6} \times \exp \left(-\frac{0.757 \mathrm{eV}}{k_{B} T}\right) \mathrm{m}^{2} \cdot \mathrm{s}^{-1} . \\
& D(\mathrm{~T})=8.99 \times 10^{-7} \times \exp \left(-\frac{0.757 \mathrm{eV}}{k_{B} T}\right) \mathrm{m}^{2} \cdot \mathrm{s}^{-1}
\end{aligned}
$$

It can be seen that the diffusion coefficients of isotopes decrease with the increasing mass. This law is consistent with the experimental results (Figure 6). The explanation of this behavior is that the lower vibrational frequency of heavier isotopes reduces the diffusion rate linearly as shown in (3). 


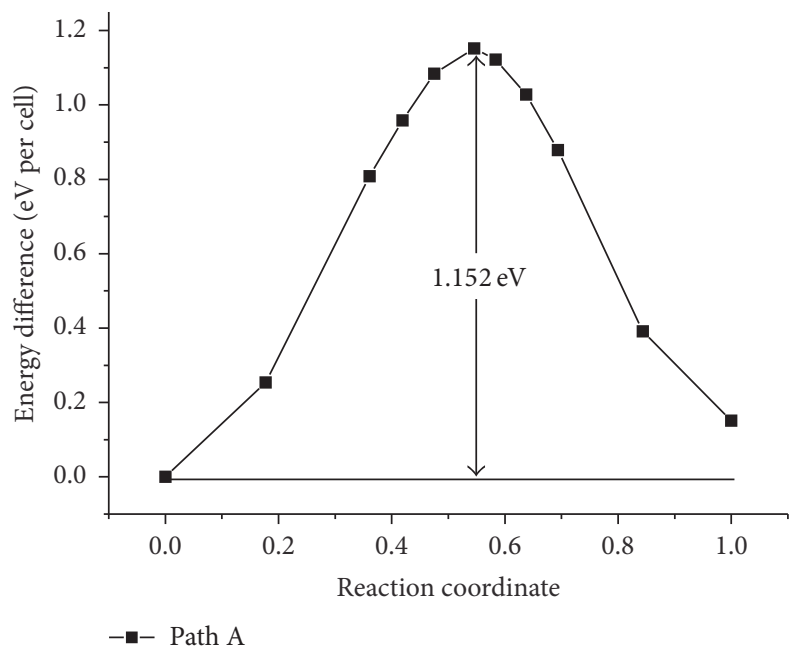

(a)

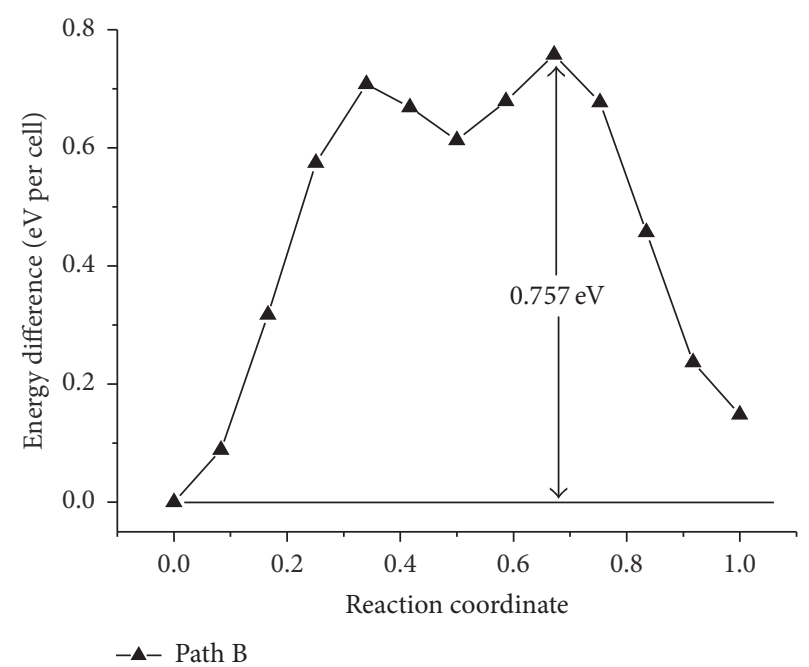

(b)

FIGURE 4: The curve for diffusion barriers of path A and path B. Owing to the symmetry of the present system, only half of the path is shown.

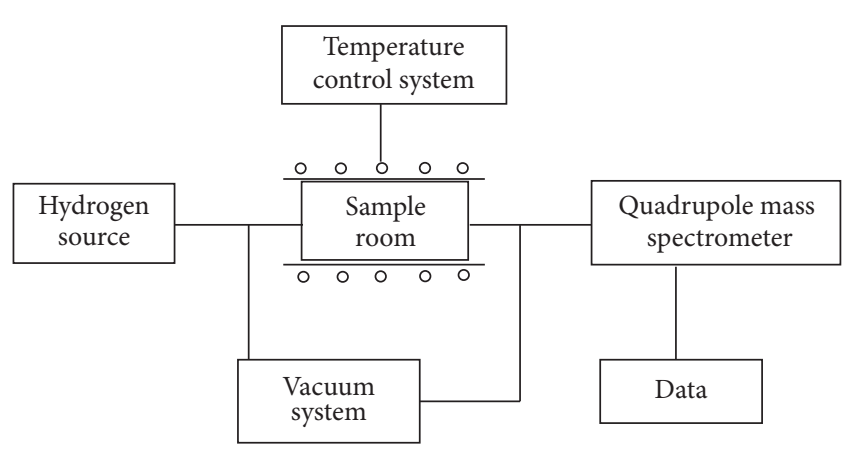

FIGURE 5: Schematic of experimental system.

\section{Conclusion}

In this study, a first-principles calculation was performed to study the diffusion behavior of tritium and its isotopes in Incoloy $800 \mathrm{H}$. Three octahedral sites and one tetrahedral site were considered, and the most stable vacancy was found to be the octahedral site surrounded by Fe atoms and Ni atoms. It was found that the diffusion of impurity atoms occurred through an indirect path via the metastable tetrahedral site and the octahedral site surrounded by Fe atoms and $\mathrm{Cr}$ atoms. The numerical results show that the diffusion activation energies of the three isotopes have the same value of approximately $0.757 \mathrm{eV}$, and the diffusion coefficients are also estimated; and $D_{0}=1.56 \times 10^{-6}, 1.10 \times 10^{-6}$, and $8.99 \times 10^{-7}\left(\mathrm{~m}^{2} / \mathrm{s}\right)$ for $\mathrm{H}, \mathrm{D}$, and T, respectively. Owing to the limitations related to experimental conditions, there are some deviations between the experimental and theoretical results.

In addition, the diffusion coefficients of tritium in Incoloy $800 \mathrm{H}$ and other alloys are still very difficult to obtain experimentally. However, the first-principles calculation provides

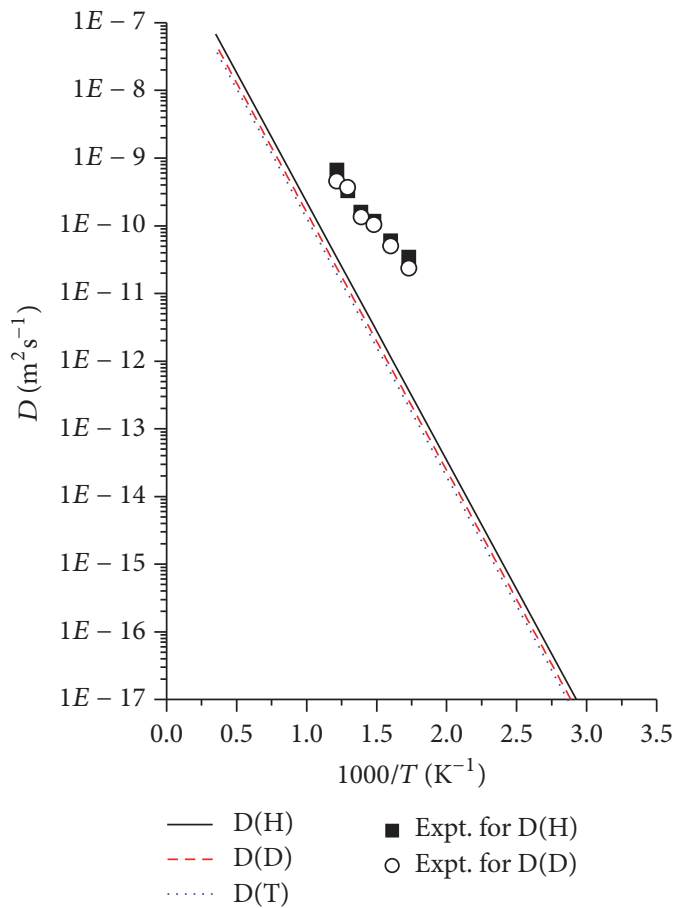

Figure 6: Computed and experimental diffusion coefficients of hydrogen isotopes.

an effective way to investigate the diffusion mechanism and related parameters. These theoretical results can be used for material selection and nuclear safety evaluation in HTR-PM.

\section{Conflicts of Interest}

The authors declare that they have no conflicts of interest. 


\section{Acknowledgments}

This work was supported by the National Science and Technology Major Project of the Ministry of Science and Technology of China (Grant no. ZX06901).

\section{References}

[1] P. Ye and X. Y. Yang, "Source and effect of Tritium from indirect closed Brayton cycle with High-Temperature Gas-Cooled Reactor," Atomic Energy Science and Technology, vol. 43, pp. 367-370, 2009.

[2] M. A. Fütterer, E. D’Agata, and X. Raepsaet, "Is tritium an issue for high temperature reactors?” Nuclear Engineering and Design, vol. 306, pp. 160-169, 2016.

[3] L. Yuanzhong and C. Jianzhu, "Fission product release and its environment impact for normal reactor operations and for relevant accidents," Nuclear Engineering and Design, vol. 218, no. 1-3, pp. 81-90, 2002.

[4] E. Wimmer, W. Wolf, J. Sticht et al., “Temperature-dependent diffusion coefficients from ab initio computations: Hydrogen, deuterium, and tritium in nickel," Physical Review B: Condensed Matter and Materials Physics, vol. 77, no. 13, Article ID 134305, 2008.

[5] B. Yang, L. G. Wang, Y. Yi, E. Z. Wang, and L. X. Peng, "Firstprinciples calculations of the diffusion behaviors of $\mathrm{C}, \mathrm{N}$ and $\mathrm{O}$ atoms in V metal," Acta Physica Sinica, vol. 64, no. 2, pp. 356361, 2015.

[6] L. F. Liu, S. Q. Zhou, Y. T. Huang, and A. R. Zhou, "Computation of diffusion activation energies of $\mathrm{C}, \mathrm{N}$ and $\mathrm{O}$ in a-Fe," Materials Review, vol. 22, no. 8, pp. 120-122, 2008.

[7] Y. Lu and P. Zhang, "First-principles study of temperaturedependent diffusion coefficients: Hydrogen, deuterium, and tritium in $\alpha$-Ti," Journal of Applied Physics, vol. 113, no. 19, Article ID 193502, 2013.

[8] M. Mantina, Y. Wang, R. Arroyave, L. Q. Chen, Z. K. Liu, and C. Wolverton, "First-principles calculation of self-diffusion coefficients," Physical Review Letters, vol. 100, no. 21, Article ID 215901, 2008.

[9] M. Mantina, Y. Wang, L. Q. Chen, Z. K. Liu, and C. Wolverton, "First principles impurity diffusion coefficients," Acta Materialia, vol. 57, no. 14, pp. 4102-4108, 2009.

[10] L. Katz, M. Guinan, and R. J. Borg, "Diffusion of H2, D2, and T2 in single-crystal Ni and Cu," Physical Review B: Condensed Matter and Materials Physics, vol. 4, no. 2, pp. 330-341, 1971.

[11] F. J. M. Boratto and R. E. Reed-Hill, "Oxygen and nitrogen diffusion in vanadium," Scripta Materialia, vol. 11, no. 12, pp. 1107-1111, 1977.

[12] T. S. Lundy and J. F. Murdock, "Diffusion of Al26 and Mn54 in Aluminum," Journal of Applied Physics, vol. 33, no. 5, pp. 1671$1673,1962$.

[13] S. J. Rothman, N. L. Peterson, L. J. Nowicki, and L. C. Robinson, "Tracer diffusion of magnesium in aluminum single crystals," Physica Status Solidi (b) - Basic Solid State Physics, vol. 63, no. 1, pp. K29-K33, 1974.

[14] T. Tanabe, S. Imoto, and Y. Miyata, "Hydrogen Permeation through Incoloy-800," Journal of Nuclear Science and Technology, vol. 16, no. 4, pp. 301-302, 1979.

[15] W. Kohn and L. J. Sham, "Self-consistent equations including exchange and correlation effects," Physical Review A, vol. 140, no. 4A, pp. A1133-A1138, 1965.
[16] G. Kresse and J. Furthmüller, "Efficiency of ab-initio total energy calculations for metals and semiconductors using a plane-wave basis set," Computational Materials Science, vol. 6, no. 1, pp. 1550, 1996

[17] G. Kresse and J. Furthmüller, "Efficient iterative schemes for ab initio total-energy calculations using a plane-wave basis set," Physical Review B: Condensed Matter and Materials Physics, vol. 54, no. 16, pp. 11169-11186, 1996.

[18] G. Kresse and J. Hafner, "Ab initio molecular dynamics for liquid metals," Physical Review B: Condensed Matter and Materials Physics, vol. 47, no. 1, pp. 558-561, 1993.

[19] G. Kresse and D. Joubert, "From ultrasoft pseudopotentials to the projector augmented-wave method," Physical Review B: Condensed Matter and Materials Physics, vol. 59, no. 3, pp. 1758$1775,1999$.

[20] J. P. Perdew, K. Burke, and M. Ernzerhof, "Generalized gradient approximation made simple," Physical Review Letters, vol. 77, no. 18, pp. 3865-3868, 1996.

[21] L. Y. Wang, "Compositions and structure feature of an Incoloy 800H alloy," Special Steel, vol. 15, no. 1, pp. 41-44, 1994.

[22] G. H. Vineyard, "Frequency factors and isotope effects in solid state rate processes," Journal of Physics and Chemistry of Solids, vol. 3, no. 1-2, pp. 121-127, 1957.

[23] R. Kutner, "Chemical diffusion in the lattice gas of noninteracting particles," Physics Letters A, vol. 81, no. 4, pp. 239240, 1981.

[24] C. Zener and C. Wert, "Interstitial atomic diffusion coefficients," Physical Review, vol. 76, no. 8, pp. 1169-1175, 1949.

[25] C. A. Wert, "Diffusion coefficient of C in $\alpha$-iron," Physical Review, vol. 79, no. 4, pp. 601-605, 1950.

[26] G. Henkelman and H. Jónsson, "Improved tangent estimate in the nudged elastic band method for finding minimum energy paths and saddle points," The Journal of Chemical Physics, vol. 113, no. 22, pp. 9978-9985, 2000. 


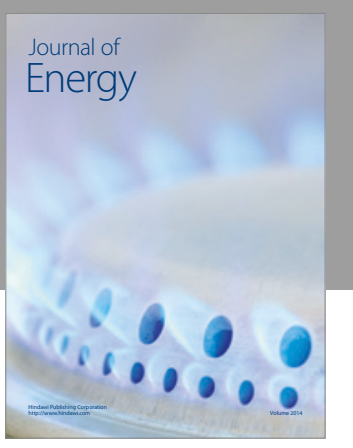

Journal of

Industrial Engineering
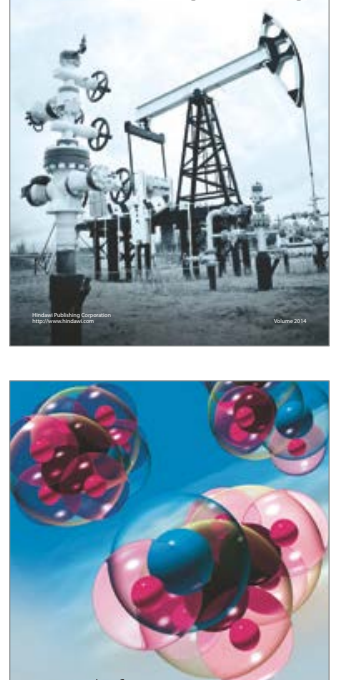

Fuels
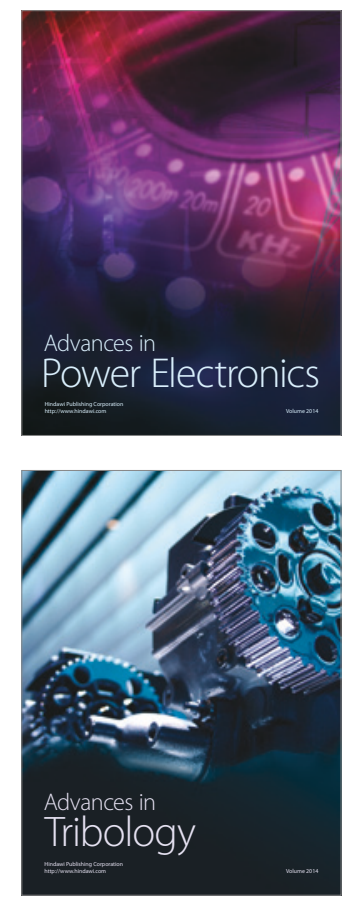
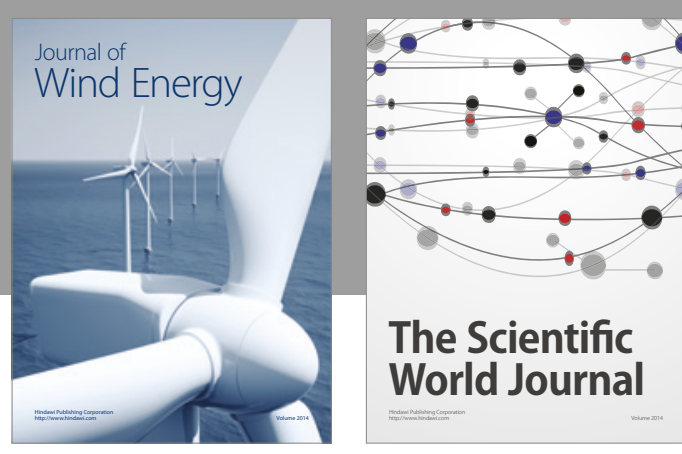

The Scientific World Journal
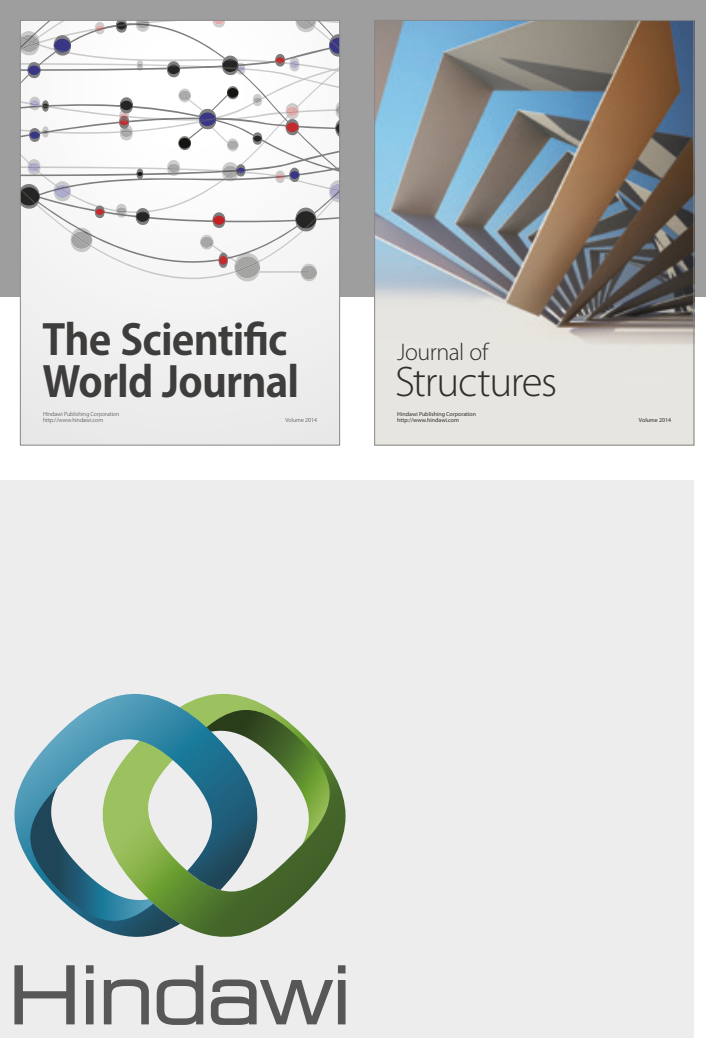

Submit your manuscripts at

https://www.hindawi.com
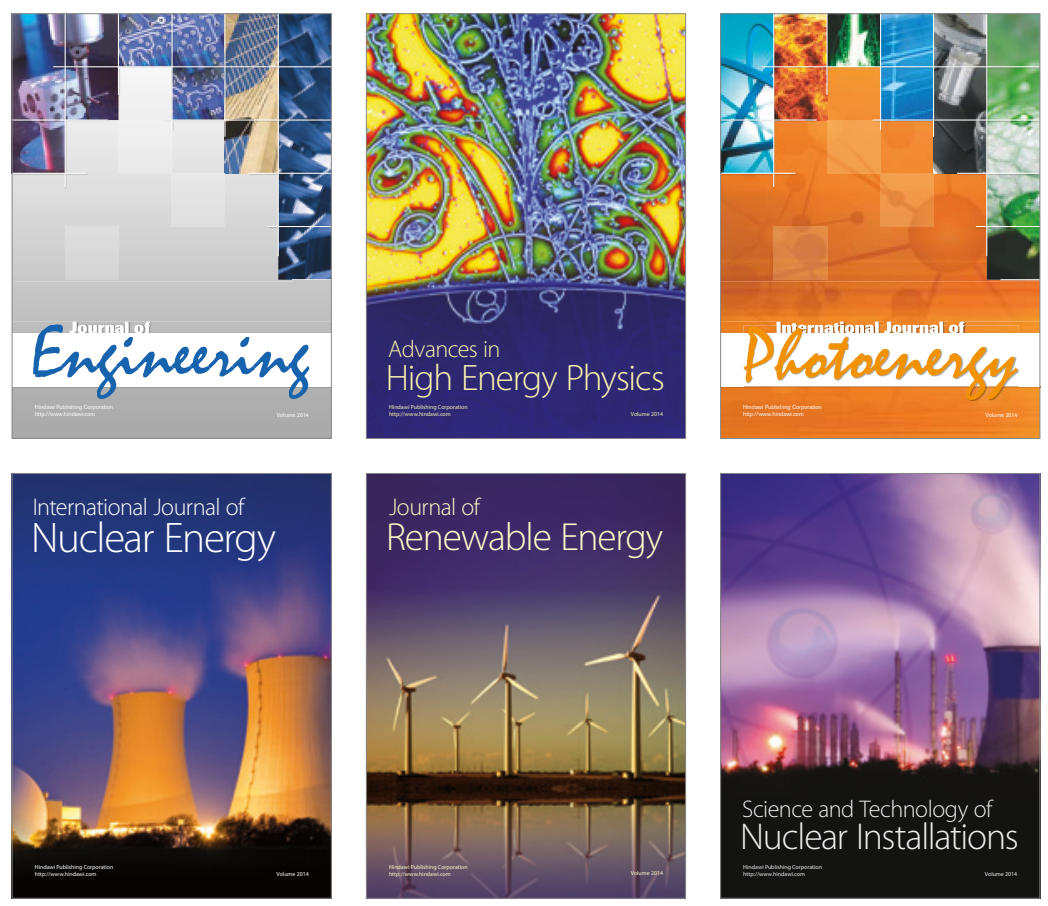

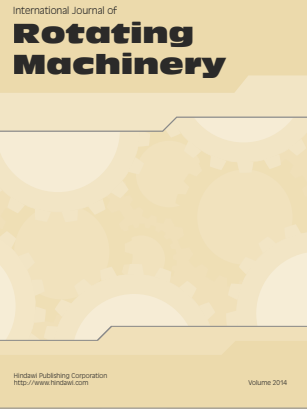

Journal of

Petroleum Engineering

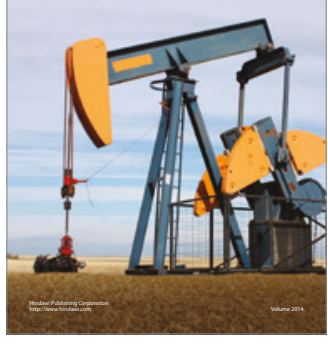

Journal of
Solar Energy
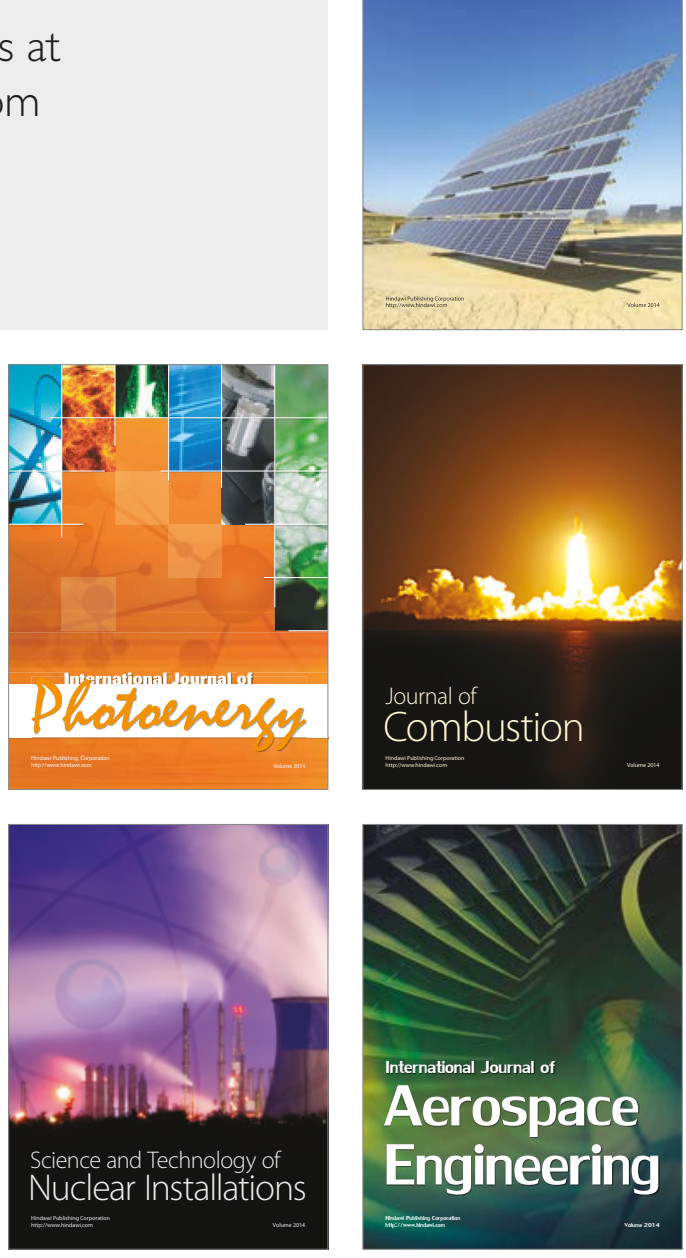